\title{
Psychiatric Comorbidity and Quality of Life in Patients with Post-Stroke Emotional Incontinence
}

\author{
Dong-Ho Choi ${ }^{1}$, Bo-Ok Jeong1', Hee-Ju Kang1', Sung-Wan Kim¹, Jae-Min Kim ${ }^{\circledR}$, Il-Seon Shin', \\ Joon-Tae Kim², Man-Seok Park², Ki-Hyun $\mathrm{Cho}^{2}$, and Jin-Sang Yoon ${ }^{1}$ \\ 1'Department of Psychiatry, Chonnam National University Medical School, Gwangju, Republic of Korea \\ 2Department of Neurology, Chonnam National University Medical School, Gwangju, Republic of Korea
}

Objective We aimed to investigate the associations of post-stroke emotional incontinence (PSEI) with various psychiatric symptoms and quality of life independent of potential covariates in survivors of acute stroke.

Methods A total of 423 stroke patients were assessed within 2 weeks of the index event. Psychiatric symptoms were assessed by the Symptom Checklist-90-Revised (SCL-90-R), which has nine domains comprising Somatization, Obsessive-Compulsive, Interpersonal Sensitivity, Depression, Anxiety, Hostility, Phobic Anxiety, Paranoid Ideation, and Psychoticism. Quality of life was measured using the World Health Organization Quality of Life abbreviated form (WHOQOL-BREF), which has four domains related to physical factors, psychological factors, social relationships, and environmental context. Associations of PSEI with scores on the SCL-90-R and WHOQOLBREF were investigated using pairwise logistic regression model adjustment for potential sociodemographic and clinical covariates.

Results PSEI was present in 51 (12.1\%) patients. PSEI was associated with the Obsessive-Compulsive, Interpersonal Sensitivity, and Hostility symptom dimensions of the SCL-90-R and with the psychological factors and social relationships domains of the WHOQOLBREF independent of important covariates including previous stroke, stroke severity, and physical disability.

Conclusion PSEI causes some aspects of psychiatric distress and negatively affects psychological and interpersonal quality of life. For patients with PSEI, special attention to psychiatric comorbidity and quality of life is needed, even in the acute stage of stroke.

Psychiatry Investig 2013;10:382-387

Key Words Stroke, Emotional lability, Psychiatric comorbidity, Quality of life.

\section{INTRODUCTION}

Emotional lability is a common syndrome of affective dysregulation caused by many neurological disorders, including stroke, multiple sclerosis, amyotrophic lateral sclerosis, and traumatic brain injury. ${ }^{1}$ Emotional lability is characterized by brief, but frequent and intense, episodes of uncontrollable crying and/or laughing. Its relationship with environmental stimuli is unclear, but these symptoms can be triggered by minor, nonspecific stimuli. Other terms that have been used to describe these emotional changes include the pseudobulbar af-

Received: January 28, 2013 Revised: April 1, 2013

Accepted: April 2, 2013 Available online: October 16, 2013

$\triangle$ Correspondence: Jae-Min Kim, MD, PhD

Department of Psychiatry, Chonnam National University Medical School, 42 Jebong-ro, Dong-gu, Gwangju 501-746, Republic of Korea

Tel: +82-62-220-6143, Fax: +82-62-225-2351

E-mail: jmkim@chonnam.ac.kr

(a) This is an Open Access article distributed under the terms of the Creative Commons Attribution Non-Commercial License (http://creativecommons.org/licenses/by$\mathrm{nc} / 3.0$ ) which permits unrestricted non-commercial use, distribution, and reproduction in any medium, provided the original work is properly cited. fect, emotionalism, pathological laughing and crying, and emotional incontinence. The prevalence of post-stroke emotional incontinence (PSEI, the term adopted here) reportedly ranges from $15 \%$ to $34 \% .^{2-6}$

PSEI is a neurological condition in which serotonergic system dysfunction is implicated as a main mechanism. ${ }^{7}$ PSEI also causes serious psychiatric complications because the symptoms are distressing and embarrassing for both patients and their families and therefore may interfere with interpersonal relationships and social functioning. ${ }^{8}$ However, few systematic studies of psychiatric symptoms in connection with PSEI have been conducted. Most previous studies focused on associations with depression, but the results were controversial. Some studies reported that PSEI is associated with an increase in depressive symptoms, ${ }^{2,6}$ while others did not find such associations. ${ }^{5,9}$ Associations with psychiatric symptoms other than depression have rarely been evaluated. Furthermore, stroke can cause poor quality of life (QOL) in survivors. ${ }^{10,11}$ However, only one study to date has investigated the 
association between PSEI and QOL, which reported that PSEI negatively affects both physical and mental aspects of health-related QOL in stroke survivors. ${ }^{12}$

Using a sample from a Korean stroke patient cohort, we aimed to investigate the associations of PSEI with various psychiatric symptoms and QOL independent of potential covariates, including stroke severity.

\section{METHODS}

\section{Participants}

This was a secondary analysis of a larger parent study investigating mental disorders in stroke survivors using a naturalistic prospective design. The detailed design and method have been published. ${ }^{11,13}$ Participants were consecutively recruited from all patients with recent ischemic stroke hospitalized within the Department of Neurology of Chonnam National University Hospital, Gwangju, Korea. Assessments for this analysis were performed 2 weeks after the stroke from 2006 to 2010 to investigate the acute consequences of stroke.

All patients with acute stroke hospitalized at the study center were approached regarding participation. The inclusion criteria were: 1) confirmed ischemic stroke by brain magnetic resonance imaging (MRI) [or computed tomography (CT) if MRI was contraindicated]; 2) an ability to complete the necessary investigations and questionnaires; and 3) the capacity to understand the objective of the study and provide informed consent. The exclusion criteria were: 1) severe physical illnesses that was life-threatening or interfering with the recovery from stroke; 2) communication difficulties due to dysphasia or dysarthria precluding informed consent and questionnaire completion; 3) any of the following comorbid neuropsychiatric conditions: dementia, Parkinson's disease, brain tumor, epilepsy, psychoses, or alcohol and substance dependence; 4) severe physical illnesses limiting movement prior to stroke; and 5) a Korean Mini-Mental State Examination (KMMSE) score of $<16 .{ }^{14}$ All participants provided written informed consent, and the study was approved by the Chonnam National University Hospital Institutional Review Board.

\section{PSEI}

PSEI was ascertained using Kim's criteria. ${ }^{5}$ Patients and their caregivers (usually a family member) were asked if the patient showed excessive and/or inappropriate crying and/or laughing compared with the premorbid state. "Inappropriateness" indicated crying or laughing that occurred while talking, listening, meeting people, or watching television and was confined to occasions that would not be expected to be particularly sad or amusing. When both the patient and caregiver agreed that the patient showed crying and/or laughing on at least three occasions, the patient was considered to have PSEI.

\section{Psychiatric symptoms}

Psychiatric symptoms were assessed by the Symptom Checklist-90-Revised (SCL-90-R). ${ }^{15}$ The SCL-90-R is a self-administered 90-item questionnaire with a five-point Likert Scale (1, not at all; 5, extremely often), which evaluates a broad range of psychological problems and symptoms of psychopathology in both psychiatric and medical settings. The test facilitates assessment of nine primary symptom dimensions comprising Somatization, Obsessive-Compulsive, Interpersonal Sensitivity, Depression, Anxiety, Hostility, Phobic Anxiety, Paranoid Ideation, and Psychoticism. The SCL-90-R has been formally standardized in Korean. ${ }^{16}$

\section{Quality of life}

QOL was measured by the World Health Organization Quality of Life abbreviated form (WHOQOL-BREF), a selfadministered 26-item questionnaire in which items are rated on a five-point scale. ${ }^{17}$ The four domains that the WHOQOLBREF evaluates are physical factors, psychological factors, social relationships, and environmental context. Raw domain scores are converted to a 0-100 scale for ease of comparison with other data sets, with higher scores indicating a better QOL. The WHOQOL-BREF has been formally standardized in Korean. ${ }^{18}$

\section{Sociodemographic and clinical covariates}

Age, gender, year of education, marital status, religious observation, current occupation, and previous history of depression or stroke were recorded according to information obtained from the participant or their caregiver, as appropriate. For this analysis, the following categories were applied: marital status (currently married or not), religion (religious observance or not), and occupation (current employed or not). Stroke severity was measured using the National Institutes of Health Stroke Scale (NIHSS). ${ }^{19}$ Its score ranges from 0 to 42 , with a higher score indicating more severe pathology. Physical disability was measured by the Barthel Index (BI). Its score ranges from 0 to 100, with a lower score indicating more severe disability. ${ }^{20}$ Cognitive function was evaluated by the KMMSE. Its score ranges from 0 to 30 , with a lower score indicating worse cognition.

\section{Statistical analyses}

Sociodemographic and clinical characteristics were compared between patients with and without PSEI using t-tests, $\chi^{2}$ tests, or Fisher's exact tests, as appropriate. Unadjusted associations of PSEI with the nine symptom dimensions of the SCL90-R and the four domains of the WHOQOL-BREF were ini- 
tially investigated using t-tests. Adjusted analyses were then conducted to investigate the significance of the associations independent of sociodemographic and clinical covariates not equally distributed $(\mathrm{p}<0.05)$ using pairwise logistic regression models. Statistical analyses were carried out using the SPSS 18.0 software.

\section{RESULTS}

\section{Recruitment and descriptive data}

Recruitment process of the participants is described in Fig-

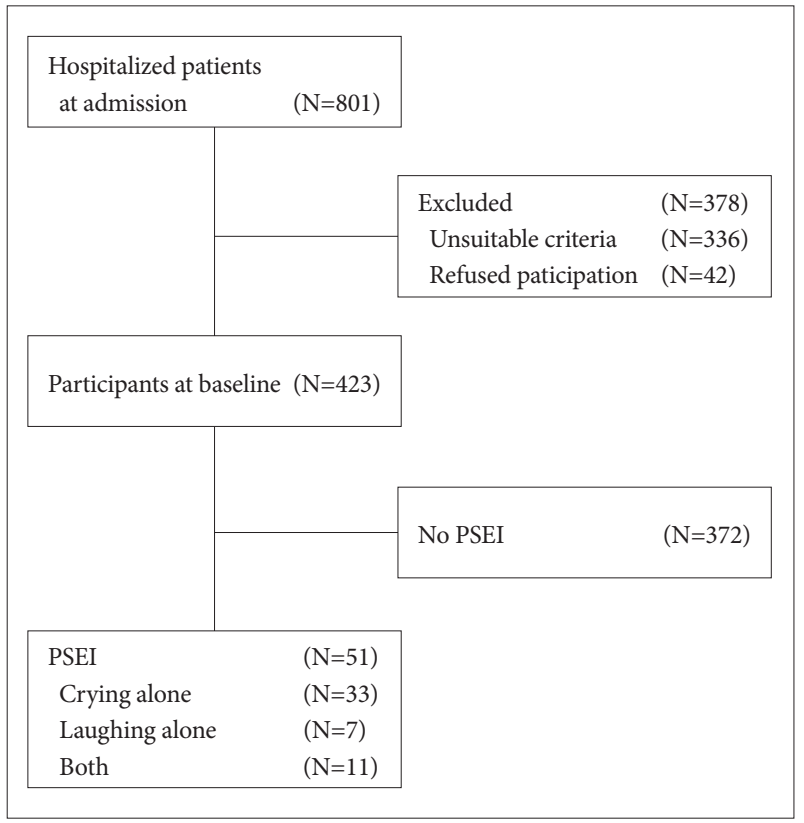

Figure 1. Recruitment process for patients with Post-stroke emotional incontinence (PSEI). ure 1. Having applied inclusion and exclusion criteria, a total of 423 stroke patients who met the inclusion and but not the exclusion criteria were recruited. The mean (SD) interview time point from stroke was 12.3 (3.0) days. The mean (SD; range) age was $64.5(10.0 ; 30-87)$ years, and $244(57.7 \%)$ patients were male. The mean (SD) duration of education was 8.6 (4.9) years, 317 (74.9\%) patients were married, 69 (16.3\%) were living alone, 232 (54.7) practiced religious observance, and 190 (44.8) had an occupation. Previous depression was reported in 17 (4.0\%) patients, and previous stroke in 35 (8.3\%). The mean (SD) NIHSS score was 3.5 (3.3), the mean (SD) BI score was 79.8 (23.2), and the mean (SD) K-MMSE score was 24.4 (4.6).

\section{Sociodemographic and clinical covariates by PSEI}

Of the 423 participants, PSEI was present in 51 (12.1\%) (33 crying alone, 7 laughing alone, and 11 both). Sociodemographic and clinical covariates according to PSEI status are shown in Table 1. Compared with patients without PSEI, those with PSEI were significantly more likely to have a previous history of stroke, higher NIHSS scores, and lower BI scores.

\section{Psychiatric symptoms according to PSEI status}

The nine symptom dimension scores on the SCL-90-R are compared according to PSEI status in Table 2. In the unadjusted analyses, patients with PSEI had significantly higher scores in seven symptom dimensions: Somatization, Obsessive-Compulsive, Interpersonal Sensitivity, Depression, Anxiety, Hostility, and Paranoid Ideation. The strengths of the associations with Obsessive-Compulsive, Interpersonal Sensitivity, and Hostility remained significant after adjustment

Table 1. Socio-demographic and clinical characteristics by post-stroke emotional incontinence (PSEI) status

\begin{tabular}{|c|c|c|c|c|}
\hline & No PSEI $(\mathrm{N}=372)$ & PSEI $(\mathrm{N}=51)$ & Statistical coefficient & p-value \\
\hline Age, mean (SD) years & $64.6(10.0)$ & $63.5(10.3)$ & $\mathrm{t}=0.780$ & 0.436 \\
\hline Gender, N (\%) male & $216(58.1)$ & $28(54.9)$ & $\chi^{2}=0.184$ & 0.668 \\
\hline Education, mean (SD) year & $8.6(4.9)$ & $8.4(5.0)$ & $\mathrm{t}=0.243$ & 0.808 \\
\hline Marital status, N (\%) married & $279(75.6)$ & $38(74.5)$ & $\chi^{2}=0.029$ & 0.864 \\
\hline Living alone, $\mathrm{N}(\%)$ yes & $62(16.7)$ & $7(13.7)$ & $\chi^{2}=0.292$ & 0.589 \\
\hline Religion, N (\%) have & $201(54.3)$ & $31(60.8)$ & $\chi^{2}=0.756$ & 0.385 \\
\hline Occupation, $\mathrm{N}(\%)$ have & $169(46.2)$ & $21(41.2)$ & $\chi^{2}=0.451$ & 0.502 \\
\hline Previous depression, N (\%) & $16(4.3)$ & $1(2.0)$ & $\chi^{2}=0.706$ & 0.370 \\
\hline Previous stroke, N (\%) & $26(7.0)$ & $9(17.6)$ & $\chi^{2}=6.713$ & 0.010 \\
\hline NIHSS, mean (SD) score & $3.3(3.1)$ & $4.9(4.0)$ & $t=-2.682$ & 0.010 \\
\hline BI, mean (SD) score & $80.8(22.8)$ & $72.7(25.7)$ & $\mathrm{t}=2.307$ & 0.022 \\
\hline MMSE, mean (SD) score & $24.5(4.5)$ & $23.8(5.1)$ & $\mathrm{t}=1.001$ & 0.317 \\
\hline
\end{tabular}

p-values using t-tests, $\chi^{2}$ tests, or Fisher's exact tests as appropriate. NIHSS: National Institutes of Health Stroke Scale, BI: barthel index, MMSE: Mini-Mental State Examination 
for previous stroke and scores on the NIHSS and BI. However, the strengths of the associations with other symptom dimensions became weaker and lost significance after adjustment.

\section{QOL according to PSEI status}

The four domain scores on the WHOQOL-BREF are compared according to PSEI status in Table 3. In the unadjusted analyses, patients with PSEI had significantly lower scores in all four domains. The strengths of the associations with the psychological factor and social relationship domains remained significant, while those with the physical factor and environment context domains lost significance after adjustment for previous stroke and scores on the NIHSS and BI.

\section{DISCUSSION}

The principal findings of this study of a sample of stroke patients were that PSEI in the acute period after stroke was associated with the Obsessive-Compulsive, Interpersonal Sensitivity, and Hostility symptom dimensions of the SCL-90-R and with the psychological factor and social relationship domains of the WHOQOL-BREF. These associations were independent of important covariates including previous stroke, stroke severity, and physical disability.

The Obsessive-Compulsive symptom domain of the SCL90- $\mathrm{R}$ is used to evaluate repetitive unwanted particular thinking or a behavior that the individual cannot stop performing.
The mechanisms underlying the obsessive-compulsive symptoms are complex, and serotonergic dysfunction is one of the main factors in the pathophysiology. Many clinical drug trials support the hypothesis that dysregulation of serotonin is involved in the development of obsessive-compulsive symptoms. Data show that serotonergic drugs are more effective than those that affect other neurotransmitter systems. ${ }^{21,22}$ In addition, the mechanism of PLC is thought to involve dysfunction of a serotonergic system; this could arise after partial destruction of the serotonergic raphe nuclei or projections from these nuclei to the hemispheres. ${ }^{23,24}$ Therefore, comorbidities of PSEI and obsessive-compulsive symptoms could be explained by the common neurobiological pathophysiology related to serotonergic dysfunction.

The Interpersonal Sensitivity symptom domain of the SCL90-R is used to assess discomfort, inappropriateness, and inferiority with respect to another individual or individuals. These are considered to be core symptoms of anxiety disorder, social phobia in particular. The biological mechanism of this disorder is serotonergic dysfunction. The identification of many serotonin receptor types has stimulated the search for the role of serotonin in the pathogenesis of anxiety disorder. The effectiveness of buspirone, a serotonin $5-\mathrm{HT}_{\mathrm{Al}}$ receptor agonist, in the treatment of anxiety disorders suggests the possibility of an association between serotonin and anxiety, ${ }^{25,26}$ and SSRIs are commonly used to treat anxiety disorders. ${ }^{27,28}$ Besides the shared a biological mechanism, the association between PSEI and interpersonal sensitivity symptoms

Table 2. Symptom dimension scores on Symptom Checklist-90-Revised by post-stroke emotional incontinence (PSEI) status

\begin{tabular}{|c|c|c|c|c|c|}
\hline Symptom dimension & No PSEI $(\mathrm{N}=372)$ & PSEI $(\mathrm{N}=51)$ & Statistical coefficient & $\mathrm{p}$-value & $\mathrm{p}^{\text {-value }}$ \\
\hline Somatization & $17.7(4.1)$ & $18.5(4.8)$ & $\mathrm{t}=-2.096$ & 0.041 & 0.069 \\
\hline Obsessive-compulsive & $12.1(2.7)$ & $13.8(3.7)$ & $\mathrm{t}=-3.294$ & 0.002 & 0.003 \\
\hline Interpersonal sensitivity & $9.0(1.7)$ & $11.0(2.8)$ & $\mathrm{t}=-2.818$ & 0.007 & 0.018 \\
\hline Depression & $17.6(5.1)$ & $18.9(7.3)$ & $\mathrm{t}=-2.172$ & 0.033 & 0.063 \\
\hline Anxiety & $11.5(2.5)$ & $12.7(3.6)$ & $\mathrm{t}=-2.099$ & 0.040 & 0.081 \\
\hline Hostility & $5.6(1.3)$ & $7.9(1.3)$ & $\mathrm{t}=-2.742$ & 0.015 & 0.012 \\
\hline Phobic anxiety & $7.3(1.6)$ & $7.9(2.4)$ & $\mathrm{t}=-1.924$ & 0.060 & 0.123 \\
\hline Paranoid ideation & $6.3(1.0)$ & $6.7(1.3)$ & $\mathrm{t}=-2.341$ & 0.023 & 0.111 \\
\hline Psychoticism & $10.2(1.3)$ & $10.4(1.2)$ & $\mathrm{t}=-0.870$ & 0.385 & 0.405 \\
\hline
\end{tabular}

*adjusted for previous stroke and scores on National Institutes of Health Stroke Scale and Barthel index

Table 3. Domain scores on World Health Organization Quality of Life-Brief form by post-stroke emotional incontinence (PSEI) status

\begin{tabular}{|c|c|c|c|c|c|}
\hline Domain & No PSEI $(\mathrm{N}=372)$ & PSEI $(\mathrm{N}=51)$ & Statistical coefficient & $\mathrm{p}$-value & p-value* \\
\hline Physical & $48.1(15.5)$ & $42.9(18.6)$ & $\mathrm{t}=2.141$ & 0.033 & 0.126 \\
\hline Psychological & $51.5(16.0)$ & $45.5(20.1)$ & $\mathrm{t}=2.422$ & 0.016 & 0.013 \\
\hline Social relationships & $56.4(11.8)$ & $50.8(12.0)$ & $\mathrm{t}=-2.218$ & 0.027 & 0.030 \\
\hline Environment & $47.5(13.6)$ & $42.8(14.3)$ & $\mathrm{t}=2.070$ & 0.043 & 0.063 \\
\hline
\end{tabular}

*adjusted for previous stroke and scores on National Institutes of Health Stroke Scale and Barthel index 
can be commonly understood by the embarrassing, abrupt nature of PSEI.

The Hostility symptom domain of the SCL-90-R is related to negative feelings and behaviors including rage, aggression, and irritability. The biological mechanisms underlying this psychopathology are also associated with serotonergic depletion, ${ }^{29,30}$ and SSRIs are commonly prescribed for these conditions. ${ }^{31}$ Patients with stroke often become easily irritated, impulsive, and angry at or aggressive toward others. The central feature of these symptoms may be described as an inability to control anger or aggression (ICAA). ICAA is not infrequent in patients with stroke and is related to serotonergic dysfunction, which is a mechanism of PSEI. ICAA and PSEI tend to co-occur and share a similar mechanism. ${ }^{32,33}$ Thus, if a patient with stroke presents with symptoms of ICAA, simultaneous evaluation for PSEI is advisable.

PSEI has been reported to be associated with an increase in depressive symptoms. ${ }^{2,6}$ In our study, the PSEI patients had no association with depression, but an association was shown in previous studies. ${ }^{5.9}$ It is difficult to distinguish crying, a symptom of PSEI, from easily weeping, a symptom of post-stroke depression (PSD), due to their similarity. From a pathophysiological perspective, we consider that PSEI is a neurological disorder directly related to serotonergic dysfunction but that PSD is psychiatric disorder with a bio-psycho-social aspect. ${ }^{34}$

Previous studies have reported that stroke survivors have low scores in all four domains of the WHOQOL-BREF.35,36 With respect to PSEI, a recent study reported that when PSEI and non-PSEI groups were compared with respect to healthrelated QOL, significant differences were found in the four domains (General Health, Social Function, Mental Health, and Role Limitations due to emotional problems) of the 36Item Short Form Health Survey. ${ }^{12}$ Similarly, in our study, the associations with the psychological and social relationship domains of the WHOQOL-BREF remained significant after adjustment for previous stroke and scores on the NIHSS and BI. Psychiatric symptoms such as interpersonal sensitivity and impulsivity, which are PSEI symptoms of an embarrassing, abrupt nature, may make personal relationships difficult and negatively influence the state of mind. These factors affect the results of QOL according to PSEI status. Thus, we propose that PSEI is a meaningful determinant of QOL in stroke patients, at least those with relatively mild physical impairment.

Our study has several clinical implications. Previous studies have reported that patients with obsessive-compulsive disorder have a worse quality of life compared with healthy controls. ${ }^{37}$ Social phobia can profoundly disrupt functioning in areas such as academic achievement and interfere with job performance and social development. ${ }^{38}$ Furthermore, patients with impulse control disorders such as pathological gambling and kleptomania have been reported to have a significantly poorer quality of life than do normal control subjects. ${ }^{39}$ In particular, patients with brain lesions have frequent and severe episodes of uncontrolled impulsivity. ${ }^{40}$ Similarly, our patients with PSEI in the acute period after stroke had high scores on the obsessive-compulsive, interpersonal sensitivity, and hostility symptom dimensions of the SCL-90-R. If patients do not receive proper treatment for PSEI after stroke, they will suffer from psychological distress, physical disability, and, ultimately, poor quality of life. Thus, administration of an appropriate pharmacological treatment and development of coping skills for social and interpersonal situations is critical for the wellbeing and quality of life of patients with PSEI.

Our study has several strengths. First, to our knowledge, previous studies focused mainly on depression related to PSEI, ${ }^{9}$ but we investigated various psychiatric symptoms related to PSEI using the SCL-90-R. Second, to diagnose PSEI, we used standardized criteria. Third, for all patients, assessments were performed 2 weeks after the stroke to investigate the consequences of stroke in the acute stage, which reduces the risk of bias and increases the potential generalizability compared with studies with more heterogeneous follow-up periods.

However, our study has several limitations. First, participants with severe cognitive impairment or aphasia who could not complete the self-rating scales or psychiatric interview were excluded from the study, and thus the generalizability of our results is limited to people with mild to moderate stroke severity without these deficits. Second, factors potentially related to PSEI such as lesion number, size, hemisphere, and location of acute infarct were not considered. ${ }^{6}$ Third, The SCL90-R is not a useful tool for detecting subtle changes in psychiatric symptoms, particularly in elderly individuals. ${ }^{41}$ Thus, other psychological tests, such as the Minnesota Multiphasic Personality Inventory (MMPI), should be used to evaluate patient symptoms in future studies. Finally, a long-term follow-up study is needed to understand the psychiatric correlates of PSEI during the chronic stage of stroke.

In conclusion, this study has shown that PSEI causes psychological distress and negatively affects psychological and interpersonal QOL. Therefore, special attention to psychiatric comorbidities and QOL is needed in patients with PSEI, even during the acute stage of stroke. PSEI is known to respond well to SSRIs. ${ }^{7}$ Introduction of appropriate pharmacological treatment as well as coping skills for social and interpersonal situations might be helpful for patients with PSEI.

\section{Acknowledgments}

This research was supported by Basic Science Research Program through the National Research Foundation of Korea (NRF) funded by the Ministry of Education, Science and Technology (2009-0087344) and by a grant of the 
Korean Health Technology R\&D Project, Ministry of Health \& Welfare, Republic of Korea (A120004).

\section{REFERENCES}

1. Arciniegas DB, Topkoff J. The neuropsychiatry of pathologic affect: an approach to evaluation and treatment. Semin Clin Neuropsychiatry 2000;5:290-306.

2. House A, Dennis M, Molyneux A, Warlow C, Hawton K. Emotionalism after stroke. BMJ 1989;298:991-994.

3. Morris PL, Robinson RG, Raphael B. Emotional lability after stroke. Aust N Z J Psychiatry 1993;27:601-605.

4. MacHale SM, O’Rourke SJ, Wardlaw JM, Dennis MS. Depression and its relation to lesion location after stroke. J Neurol Neurosurg Psychiatry 1998;64:371-374

5. Kim JS, Choi-Kwon S. Poststroke depression and emotional incontinence: correlation with lesion location. Neurology 2000;54:1805-1810.

6. Tang WK, Chan SS, Chiu HF, Ungvari GS, Wong KS, Kwok TC. Emotional incontinence in Chinese stroke patients--diagnosis, frequency, and clinical and radiological correlates. J Neurol 2004;251:865-869.

7. Wortzel HS, Oster TJ, Anderson CA, Arciniegas DB. Pathological laughing and crying: epidemiology, pathophysiology and treatment. CNS Drugs 2008;22:531-545.

8. Andersen G, Vestergaard K, Riis JO. Citalopram for post-stroke pathological crying. Lancet 1993;342:837-839.

9. Calvert T, Knapp P, House A. Psychological associations with emotionalism after stroke. J Neurol Neurosurg Psychiatry 1998;65:928-929.

10. Viitanen M, Fugl-Meyer KS, Bernspång B, Fugl-Meyer AR. Life satisfaction in long-term survivors after stroke. Scand J Rehabil Med 1988; 20:17-24.

11. Jeong BO, Kang HJ, Bae KY, Kim SW, Kim JM, Shin IS, et al. Determinants of quality of life in the acute stage following stroke. Psychiatry Investig 2012;9:127-133.

12. Chen YK, Wong KS, Mok V, Ungvari GS, Tang WK. Health-related quality of life in patients with poststroke emotional incontinence. Arch Phys Med Rehabil 2011;92:1659-1662.

13. Kim JM, Stewart R, Bae KY, Kim SW, Kang HJ, Shin IS, et al. Serotonergic and BDNF genes and risk of depression after stroke. J Affect Disord 2012;136:833-840.

14. Kang YW, Na DL, Han SH. A validity study on the Korean Mini-Mental State Examination (K-MMSE) in dementia patients. J Korean Neurol Assoc 1997;15:300-308.

15. Derogatis LR. SCL-90(Revised) Manual I Clinical Psychometrics Research Unit. Baltimore: University School of Medicine; 1977.

16. Kim KI, Kim JH, Won HT. Korean Manual of Symptom Checklist90-revision. Seoul: Chung Ang Aptitude Publishing Co.; 1984.

17. Development of the World Health Organization WHOQOL-BREF quality of life assessment. WHOQOL Group. Psychol Med 1998;28: 551-558.

18. Min SK, Kim KI, Lee CI, Jung YC, Suh SY, Kim DK. Development of the Korean versions of WHO Quality of Life scale and WHOQOLBREF. Qual Life Res 2002;11:593-600.

19. Kasner SE, Chalela JA, Luciano JM, Cucchiara BL, Raps EC, McGarvey $\mathrm{ML}$, et al. Reliability and validity of estimating the NIH stroke scale score from medical records. Stroke 1999;30:1534-1537.

20. Mahoney FI, Barthel DW. Functional evaluation: the Barthel index. Md State Med J 1965;14:61-65.

21. Greist JH, Jefferson JW, Kobak KA, Katzelnick DJ, Serlin RC. Efficacy and tolerability of serotonin transport inhibitors in obsessive-compulsive disorder. A meta-analysis. Arch Gen Psychiatry 1995;52:53-60.

22. Pigott TA, Seay SM. A review of the efficacy of selective serotonin reuptake inhibitors in obsessive-compulsive disorder. J Clin Psychiatry 1999; 60:101-106.

23. Andersen G, Ingeman-Nielsen M, Vestergaard K, Riis JO. Pathoanatomic correlation between poststroke pathological crying and damage to brain areas involved in serotonergic neurotransmission. Stroke 1994; 25:1050-1052.

24. Kim SW, Shin IS, Kim JM, Lim SY, Yang SJ, Yoon JS. Mirtazapine treatment for pathological laughing and crying after stroke. Clin Neuropharmacol 2005;28:249-251.

25. Tancer ME, Uhde TW. Role of serotonin drugs in the treatment of social phobia. J Clin Psychiatry 1997;58(Suppl 5):50-54

26. Laakmann G, Schüle C, Lorkowski G, Baghai T, Kuhn K, Ehrentraut S. Buspirone and lorazepam in the treatment of generalized anxiety disorder in outpatients. Psychopharmacology (Berl) 1998;136:357-366.

27. Davidson JR. Pharmacotherapy of social anxiety disorder: what does the evidence tell us? J Clin Psychiatry 2006;67(Suppl 12):20-26.

28. Stein MB, Stein DJ. Social anxiety disorder. Lancet 2008;371:1115-1125.

29. Goldstein M. Brain research and violent behavior. A summary and evaluation of the status of biomedical research on brain and aggressive violent behavior. Arch Neurol 1974;30:1-35.

30. Davidson RJ, Putnam KM, Larson CL. Dysfunction in the neural circuitry of emotion regulation--a possible prelude to violence. Science 2000;289:591-594.

31. Grant JE, Potenza MN. Impulse control disorders: clinical characteristics and pharmacological management. Ann Clin Psychiatry 2004;16: 27-34.

32. Paradiso S, Robinson RG, Arndt S. Self-reported aggressive behavior in patients with stroke. J Nerv Ment Dis 1996;184:746-753.

33. Kim JS, Choi S, Kwon SU, Seo YS. Inability to control anger or aggression after stroke. Neurology 2002;58:1106-1108.

34. Kang HJ, Kim SW, Kim JM, Shin IS, Yoon JS. Pathogenesis of poststroke depression: a bio-psycho-social integrative model. J Korean Neuropsychiatr Assoc 2011;50:347-353.

35. Gupta A, Deepika S, Taly AB, Srivastava A, Surender V, Thyloth M. Quality of life and psychological problems in patients undergoing neurological rehabilitation. Ann Indian Acad Neurol 2008;11:225-230.

36. Raju RS, Sarma PS, Pandian JD. Psychosocial problems, quality of life, and functional independence among Indian stroke survivors. Stroke 2010;41:2932-2937.

37. Moritz S, Rufer M, Fricke S, Karow A, Morfeld M, Jelinek L, et al. Quality of life in obsessive-compulsive disorder before and after treatment. Compr Psychiatry 2005;46:453-459.

38. Sadock BJ, Sadock VA. Anxiety Disorder-Specific Phobia and Social Phobia. In: Sadock BJ, Sadock VA, editors. Synopsis of Psychiatry, Behavioral Sciences/Clinical Psychiatry. 10th ed. Baltimore: Lippincott Williams \& Wilkins, 2007, p.597-604.

39. Grant JE, Kim SW. Quality of life in kleptomania and pathological gambling. Compr Psychiatry 2005;46:34-37.

40. Sadock BJ, Sadock VA. Impulse-Control Disorders Not Elsewhere Classified. In: Sadock BJ, Sadock VA, editors. Synopsis of Psychiatry, Behavioral Sciences/Clinical Psychiatry. 10th ed. Baltimore: Lippincott Williams \& Wilkins, 2007, p.773-776.

41. Schmitz N, Hartkamp N, Kiuse J, Franke GH, Reister G, Tress W. The Symptom Check-List-90-R (SCL-90-R): a German validation study. Qual Life Res 2000;9:185-193. 